\title{
Object Classification Using Hybrid Fiber Optical Force/Proximity Sensor*
}

\author{
Jelizaveta Konstantinova ${ }^{1}$, Giuseppe \\ Cotugno ${ }^{1}$, Agostino Stilli \\ School of Eng. \& Materials Science \\ Queen Mary University of London \\ London, United Kingdom \\ j.konstantinova@qmul.ac.uk
}

\author{
Yohan Noh \\ Department of Informatics \\ King's College London \\ London, United Kingdom
}

\author{
Kaspar Althoefer \\ Advanced Robotics @ Queen Mary (ARQ) \\ Faculty of Engineering Science \\ Queen Mary University of London \\ London, United Kingdom
}

\begin{abstract}
Intelligent perception to determine the physical interaction between robotic hands and the environment is a topic of great interest for the sensing and robotics communities. Sensor information on object stiffness and associated object deformation is essential to plan and execute stable and tight grasps. This paper proposes a novel robot-finger-integrated perception sensor to estimate the physical interaction with objects. The sensing principle of our combined force and proximity sensor is based on light intensity modulation, involving fiber optics technology to measure the proximity between robot fingers and object during approach as well as to detect normal and lateral forces. In order to distinguish between hard and soft (deformable) objects a Support Vector Machine (SVM) is employed to classify the handled object's stiffness based on the measured force and proximity data. The classifier does not require prior knowledge of the objects and achieves $87 \%$ classification accuracy on a set of household objects with different mechanical characteristics.
\end{abstract}

Keywords-fiber optical sensing, force sensing, proximity sensing, stiffness classification.

\section{INTRODUCTION}

It is well known that the quality of grasping and manipulation tasks performed by multi-fingered robotic hands can be improved with the use of tactile and proprioceptive sensing [2]. Such sensory information can be used to obtain an understanding regarding the geometry, position, orientation and stiffness of the handled object, allowing us to evaluate and improve the quality of grasping. Visual information is often used to estimate the shape and location of an object, as well as to create a virtual representation that can be used for grasp planning [1]. Visual recognition, however, provides little information on the mechanical characteristics of the object, such as stiffness. Without this knowledge, it can be extremely challenging to grasp fragile or deformable objects, such as food or clothing, as it is often difficult to determine whether the applied forces deform or, even, damage the object.

To grasp deformable objects, a robotic system should be equipped with "physical interaction" sensors installed on the gripper and appropriate perception intelligence using the sensor data to guide the grasping. Robotic grippers equipped with tactile sensors are becoming increasingly popular. Although force and tactile sensors are steadily improving, there is still the need for further hardware development and enhanced algorithms that enable the generalization of perceived information [2].

*The research leading to these results has received funding from the European Community's Seventh Framework Programme under grant agreement no. 610532, SQUIRREL, and from the European Unions Horizon 2020 research and innovation programme under grant agreement no. 287728 , FourByThree.

${ }^{1}$ These authors have equally contributed to the manuscript.

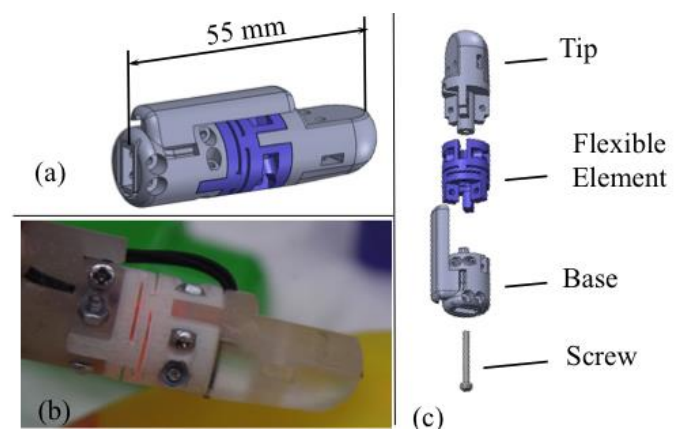

Fig. 1. Integrated fingertip sensor: a) CAD model; b) prototype implemented using three-dimensional printing; c) exploded view of the sensor structure.

There is a category of model-based control algorithms for deformable object grasping strongly relying on advanced sensing and computation capabilities and precise knowledge of the objects used, usually acquired through repeated exploratory grasps to identify the mechanical characteristics of the objects in question [3], [4]. These requirements are very specific, and often limit the use of these control algorithms to a specific application domain. Other approaches make use of vision systems to aid object recognition and grasping [5]. The disadvantage of this approach is the complexity of the models and exorbitant computational requirements, as vision processing is a difficult resource-intensive operation. More recent work [6] applies machine learning techniques to a set of features extracted from raw data of the interaction between the object and sensors. Similarly, using a Support Vector Machine (SVM), our method classifies objects into hard and soft classes based on the object's deformability features as determined from proximity, torque and force features acquired during the initial interaction with the object. This method does not require any prior knowledge of the manipulated objects and produces robust classification thanks to the fusion of different sensory data. The contribution of this work is as follows: 1) we present the design of the integrated fingertip tactile sensor for grasping; 2) we present the classification algorithm for the integrated sensor to distinguish between hard and deformable objects.

\section{SENSOR DESIGN}

The presented sensor is using optical fibers that detect light intensity at its sensing elements (Figures 1 and 2). The displacement of a non-deformable surface painted with a thin layer of reflective silver paint is used to change the light intensity; the light intensity is modulated as a function of the pressure applied to the sensing element. In addition, two 
proximity sensors are embedded in the fingertip structure. Our proximity sensor comprises a couple of optical fibers and estimates the distance to an object evaluating the amount of light that is reflected back by that object. The entire structure of the sensor integrated with the robotic fingertip can be 3D printed. The sensor can be used for any robotic hand, as only the configuration of the connecting element need to be modified. The design of our integrated hybrid fingertip sensor is shown in Figure 1. As it can be seen, from the exploded view, the sensor is composed of four main elements. The tip of the sensor has the shape of a human fingertip, and is acting as a contact surface with an object. Two proximity sensors are embedded in the tip of the fingertip. The second part of the fingertip sensor is a flexible cantilever structure (supported beam) that is used to detect normal force and lateral moments based on the deflection in directions normal to the finger's longitudinal axis. The third element of the sensor is a base that connects the fingertip with the rest of the robotic finger. Three pairs of optical fibers are embedded in the base element to detect the deflection of the flexible cantilever structure above. The fiber tips are directed to the reflective surface applied to the top structure (Figure 2). The final element of the sensor structure is a metal screw to protect the sensor from excessive loads, by limiting the deflection; this screw is inserted in the center of the sensor to limit the bending of the flexible element.

\section{A. Force and Torque Sensing}

The flexible structure of the integrated fingertip sensor is used to detect three-axis force/torque signals. The deflection of the cantilever structure is causing the displacement of the reflective surface in respect to the tips of the pairs of the optical fibers. Figure 3 shows finite element simulations of applied normal force and lateral moments. The design of the flexible structure is based on the previous work of authors, where the similar cantilever type structure is used to measure three-axial forces for cardiac catheter [7]. The above work describes the calibration of light intensity modulation, as well as modeling of sensor response. As the dimensions and configuration of the flexible cantilever structure are different in our case, the calibration process was repeated. It was found that the fingertip sensor measures normal force up to $4.5 \mathrm{~N}$. The lateral torque values (around the $x$ - and y-axes) go up to $\pm 18 \mathrm{~N} / \mathrm{mm}$. Using nylon to print the flexible cantilever structure proved to lead to low hysteresis and high robustness.

\section{B. Proximity Sensing}

Typically, an external vision system is used to detect the location of a target object for grasping, and to estimate its position inside the hand when the grasp is complete. However, in the scenario, when the grasp is executed wrongly, fingers can occlude the object, thus limiting the possibility to adjust the grasp. Proximity sensors are used to measure the distance to the object, when the fingers are ready to grasp, when in hand manipulation or grasp adjustment is required. As shown in Figure 2, the integrated fingertip sensor is equipped with two proximity sensors. Depending on the type of grasp, and the orientation of the finger relative to the object, either single pad or tip proximity sensor or both are used to measure the distance to the object. The distance to an object is estimated using the amount of light reflected back from the object.

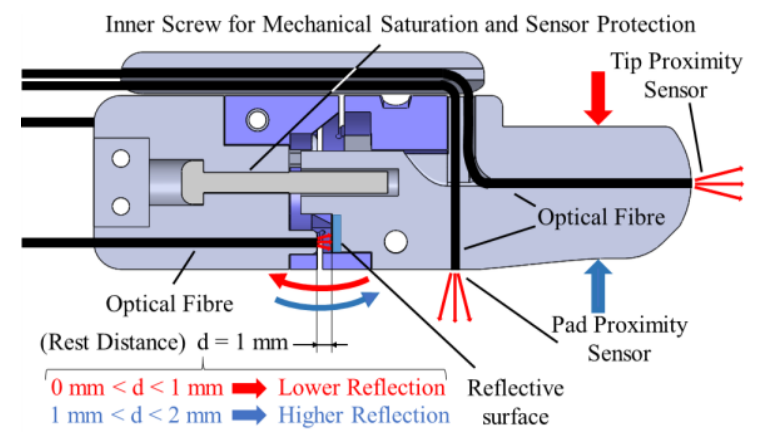

Fig. 2. Cross-section of the integrated fingertip sensor; red and blue arrows show sensor behavior for deflections in different direction.
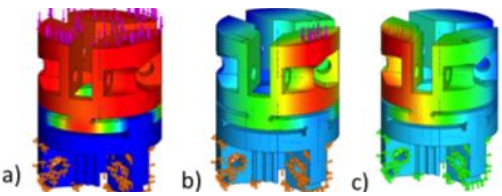

Fig. 3. Simulations of applied load to the flexible structure: a) normal force, b) and c) lateral torques.

Our proximity sensors can measure distances of up to 30 mm depending on the reflective properties of an object [8]. In previous work, the authors described the calibration method that uses an external camera to estimate object surface properties for improved distance estimates [8]. This allowed us to improve the accuracy of distance estimation (mean error: below $2 \mathrm{~mm}$ ).

\section{OBJeCt ClassificATION ALgORItHM}

\section{A. Experimental setting}

In order to design and experimentally validate the classification method, experimental trials were conducted with a range of objects (Figure 4a), which can be classified as hard or soft. The training set of objects had six hard and five soft objects. In case when the objects deformation during pressure was below $3 \mathrm{~mm}$, the object was tagged as hard. In order to perform probing motion, two sensors were mounted on the Baxter robot parallel gripper, as shown in Figure $4 \mathrm{~b}$. Five grasping trials were performed for each object. To train and test the classifier, the normal force, the lateral torques and the proximity values were obtained from each trial. The magnitude of lateral moment was used in the classification algorithm. The sensor data was filtered with a median filter of size two; it was normalized and de-trended by removing the mean value. The proximity data was processed to obtain a binary output. If the proximity sensor is triggered when approaching the object, the output was marked as 1 and the force and torque data is considered for learning. If the object is too distant, the output of the sensor is marked as 0 and the respective data is ignored. In this work, normal force, torque and proximity information are merged to help the classification. The data was preprocessed using Matlab 2016b, while the classification was performed in Weka 3.8.

\section{B. Design of the Algorithm}

The first step required for implementing a successful learning technique is to define a set of features of the data that can discriminate the data between the two classes. The input 
data is a series of $N$-by-three sequences, where $N$ is the number of points in a trial, for force, proximity and torque observations. Two features were extracted: the standard deviation of the force and torque data when the finger is in contact with the object. It is suggested that the force/torque response of a hard object is mostly steady over time. Instead, a soft object has a variable response, which depends on the finger's indentation, due to the deformability of the object's surface. As shown in the classification results in Figure 5, the use of standard deviation as learning features simplified a large set of points into a linearly separable dataset. The problem of hard/soft object classification was resolved using SVM. Such technique is well established, and is well suited for binary classification. As the data is linearly separable, the SVM can be trained using a linear kernel. In this case a linear decision threshold, which requires just three parameters, adequately separated the data. The classifier has the following form:

$$
\begin{gathered}
f(x)=-5.2629 F-2.9108 T+2.38, \\
f(x)>0 \rightarrow \text { hard, } \\
f(x)<0 \rightarrow \text { soft },
\end{gathered}
$$

where $F$ and $T$ are the force and torque value respectively.

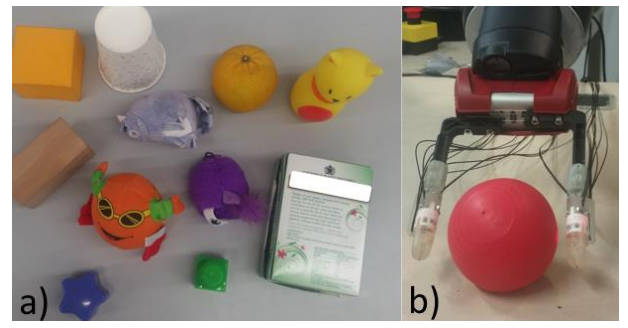

Fig. 4. Experimental setting: a) set of objects; b) executed grasp for object classification.

\section{Classification Results}

To evaluate the results of the classification, two more models were trained and used for comparison: a voted perceptron and the ZeroR classifier. The latter assumes that all instances belong to the majority class and is generally used as reference for comparison. All models were assessed with a 10fold cross-validation. The trained SVM correctly classified $87.3 \%$ of the data, as it is shown in Figure 5, and performed better than the perceptron (69\%) and the ZeroR (63.6\%). In addition, the classification precision, the recall and the area of the Receiver Operating Characteristic (ROC) curve were evaluated to compare the accuracy of the classification for all models (Table I). The precision is ratio of correct guesses on the total number of guesses for each class. The recall is the ratio of correct guesses of one class over the total number of correct guesses. The area of the ROC is an index, which indicates how well the data is separated into classes. Although the voted perceptron can discriminate linearly separable data, its performance is poorer than SVM as its algorithm, does not consider the margin to the samples.

\section{DisCUSSION AND CONCLUSIONS}

In this work we present the design of an integrated fingertip sensor that is based on a fiber optical measurement principle designed to perform intelligent grasping of a wide range of objects, including hard non-deformable and soft deformable objects. An SVM classifier, using as the input data from the integrated fingertip sensors, classifies objects into soft and had based on their deformability. The performance of the proposed classifier is compared against a baseline classifier and a perceptron and it is found to be more accurate. The proposed sensor and classifier allow planning an adequate grasping strategy after single-contact probing to build up classification features. In the future, we plan to detect the exact stiffness without the need of an exploration by using a Deep Convolutional Neural Network.

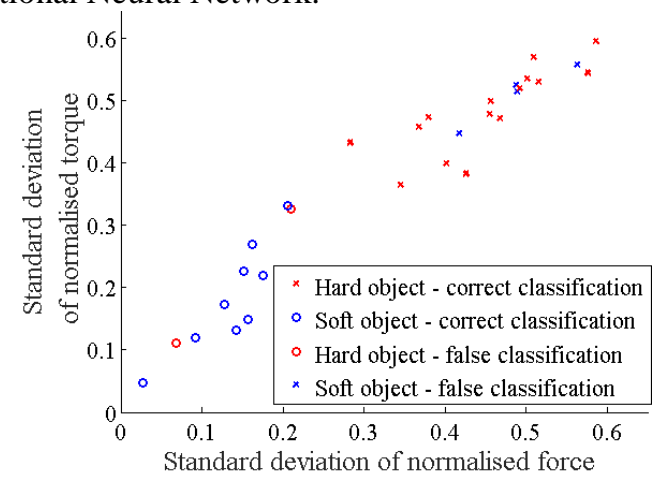

Fig. 5. Classification results of hard and soft objects using SVM.

TABLE I. COMPARISON OF CLASSIFICATION METRICS

\begin{tabular}{|c|c|c|c|c|}
\hline & Classification & Precision & Recall & ROC \\
\hline \multirow{2}{*}{$\begin{array}{c}\text { Hard } \\
\text { object }\end{array}$} & ZeroR & 0.636 & 1 & 0.464 \\
\cline { 2 - 5 } & Perceptron & 0.688 & 0.943 & 0.825 \\
\cline { 2 - 5 } & SVM & 0.889 & 0.914 & 0.857 \\
\hline \multirow{2}{*}{$\begin{array}{c}\text { Soft } \\
\text { object }\end{array}$} & ZeroR & 0 & 0 & 0.464 \\
\cline { 2 - 5 } & Perceptron & 0.714 & 0.25 & 0.825 \\
\cline { 2 - 5 } & SVM & 0.842 & 0.800 & 0.857 \\
\hline
\end{tabular}

\section{REFERENCES}

[1] F. F. Khalil and P. Payeur, "Dexterous Robotic Manipulation of Deformable Objects with Multi-Sensory Feedback - a Review," Robot Manip. Trends Dev., pp. 587-621, 2010.

[2] J. Tegin and J. Wikander, "Tactile sensing in intelligent robotic manipulation- a review," Ind. Robot An Int. J., vol. 32, pp. 64-70, 2005.

[3] K. B. Shimoga and A. A. Goldenberg, "Soft Robotic Fingertips: Part I: A Comparison of Construction Materials," Int. J. Rob. Res., vol. 15, no. 4, pp. 320-334, 1996.

[4] A. Delgado, C. A. Jara, D. Mira, and F. Torres, "A tactile-based grasping strategy for deformable objects' manipulation and deformability estimation," 2015 12th International Conference on Informatics in Control, Automation and Robotics,2015, vol.2, pp.369

[5] F. Sun, C. Liu, W. Huang, and J. Zhang, "Object Classification and Grasp Planning Using Visual and Tactile Sensing," IEEE Trans. Syst. Man, Cybern. Syst., vol. 46, no. 7, pp. 969-979, 2016.

[6] M. V. Liarokapis, B. Calli, A. J. Spiers, and A. M. Dollar, "Unplanned, model-free, single grasp object classification with underactuated hands and force sensors," in IEEE International Conference on Intelligent Robots and Systems, 2015, vol. 2015-Decem, pp. 5073-5080.

[7] Y. Noh, H. Liu, S. Sareh, D. S. Chathuranga, H. Wurdemann, K. Rhode, and K. Althoefer, "Image-based optical miniaturized three-axis force sensor for cardiac catheterization," IEEE Sens. J., vol. 16, no. 22, pp. 7924-7932, 2016.

[8] J. Konstantinova, A. Stilli, A. Faragasso, and K. Althoefer, "Fingertip proximity sensor with realtime visual-based calibration," in IEEE International Conference on Intelligent Robots and Systems, 2016, vol. 2016-Novem, pp. 170-175. 